\title{
Joint Commission Preparation: An Amazing Race
}

\author{
Elizabeth DiPietro ${ }^{*}$, Paula Reber ${ }^{2}$ and Tami Swearingen ${ }^{3}$ \\ ${ }^{1}$ Pediatric Clinical Nurse Specialist, Kennedy Krieger Institute, Baltimore, Maryland, USA \\ ${ }^{2}$ Nurse Educator, Kennedy Krieger Institute, Baltimore, Maryland, USA \\ ${ }^{3}$ Senior Vice President, Nursing and Patient Services, Kennedy Krieger Institute, Baltimore, Maryland, USA
}

\begin{abstract}
Received: November 11, 2016; Accepted: December 22, 2016; Published: January 25, 2017
*Corresponding author: Elizabeth DiPietro, Pediatric Clinical Nurse Specialist, Kennedy Krieger Institute, Baltimore, Maryland, USA; E-mail: dipietroe@ kennedykrieger.org
\end{abstract}

The Kennedy Krieger Institute, located in Baltimore, Maryland, is an internationally recognized institution dedicated to improving the lives of individuals with disorders of the brain, spinal cord, and musculoskeletal system. Our Pediatric Inpatient Unit focuses on pediatric patients with a variety of rehabilitative needs such as brain injury, spinal cord injury, chronic pain, complex medical conditions, post-orthopedic surgery, and severe feeding difficulties. The inpatient hospital is licensed for seventy beds and employs registered nurses and nurse technicians who provide twenty four hour direct, family centered care, an all-encompassing responsibility. Although Joint Commission preparedness is an ongoing process, an upcoming triennial survey still requires planning, preparation, and education. To plan for our Joint Commission survey, a creative and innovative approach was crucial. Furthermore, the staff educators wanted to ensure the education went beyond regulatory compliance. Our goal was not simply situational awareness of what is required during the actual survey in order to obtain accreditation but to make an impact on really understanding the Joint Commission regulations and National Patient Safety Goals and ensure the delivery of safe, high quality patient care.

Accreditation by the Joint Commission, an organization that certifies healthcare organizations in the United States, signifies that the organization provides safe and effective healthcare and has met certain performance standards. These standards are put into place in order to maintain or improve healthcare delivery for all individuals, across all settings. The interpretation, practical clinical application, and dissemination of these benchmarks to the nursing staff are the responsibility of the nurse educators.

Mindful of our objectives, the necessary education to be implemented needed in an inventive and creative manner in order to engage the nursing staff and maintain everyone's interest. A dull, uninteresting in-service or emails with information would be ineffective and most likely be ignored or overlooked. We needed a learning plan where the nursing staff would be motivated by a fun and friendly competition, while fully realizing the importance of the program. According to Knowles (1998) adult learners have a "need to know," to know why they need to learn certain information, and they are "motivated" to learn certain information based on both external factors (salary, tangible items) and internal factor (improved job satisfaction).
Furthermore, adult learners want to learn information that is relevant and directly applicable to their work. They also want the material to be presented in a time efficient method. The implementation of electronic learning methods such as emailed education and online testing and quiz makers recognizes these needs by delivering the educational materials in a clear concise manner that can be accessed when the adult learner is available to learn, not when the facilitator is ready to teach. This promotes flexible and self-directed learning, extremely beneficial when the group of learners is unable to assemble at the same time in a classroom setting, such in the case of registered nurses and nurse technicians who work a variety of shifts. Electronic learning supports the adult learning theory that the nurse educator is more of a facilitator as opposed to a distributor of education (Magnusson 2006).

Equipped with this information, the nurse educators developed a horse race theme, modeled after the nationally recognized Baltimore Preakness Horse Race, one of the three races in the Triple Crown of Thoroughbred Horse Racing, to educate nursing staff on the Joint Commission standards. The nursing staff members were divided into six teams, consisting of both registered nurses and nurse technicians. Each team was assigned a "team jockey" and given a wooden horse to decorate in an assigned color. The teams were very creative with their decorations and horse names (Anita Winner, Pretty Pink Star, Code Blue, Green Emerald, Fire Ball, and Miss Frizzle). To serve as a visual reminder for each team's participation and progress, a simulated horse track was created and placed in the nursing break room.

The nurse educators then developed five biweekly educational challenges based on Joint Commission standards and regulations. The first four topics were 1-Medication Management, including two patient identifiers, safe medication preparation, approved abbreviations, therapeutic duplication, and oxygen cylinder segregation; 2- Safety, Infection Control and Environment of Care; 3-Patient Care Specifics, including the National Patient Safety Goals; and 4-Restraint Management, including provider order requirements, patient safety precautions, and documentation standards. The last biweekly educational challenge was a review of all the topics to reinforce comprehension and clinical application. The educational challenges were emailed in a PowerPoint format 
incorporating both an instructive method and a caricature horse theme. To measure learning and understanding of each topic an electronic test was also included. In addition, mock onsite surveys were conducted during each biweekly session to further validate understanding.

Results were shared every two weeks to provide immediate feedback and recognize each team's progress. All participants were acknowledged and given a token incentive, such as a pocket hand sanitizer. At the completion of all of the challenges, each member of the team with the most participation was recognized and presented with a gift card. In addition, all registered nurses and nurse technicians who achieved a $100 \%$ test score on all of the biweekly challenges received a participation prize.

The ten week long educational session was successful, resulting in a $90 \%$ average participation rate amongst the 86 nursing staff members and an $88 \%$ test average. The overall feedback included the following statements: "the program presentation was in an easy to learn formatting", "convenient," (available when the learner was ready to learn), "applicable to current practice", "reinforced safe patient care," and "highlighted new patient safety goals, "while providing a fun and slightly competitive game between co-workers.
The Joint Commission triennial survey was conducted approximately five weeks after the conclusion of the educational sessions and confirmed our hospital meets the performance standards that promote safe and effective care of the highest quality and value. In fact, some projects were specifically recognized as best practices that were shared through The Joint Commission Leading Practice Library. Successful accreditation is significant as the Joint Commission standards are "the basis of an objective evaluation process that help health care organizations measure, assess and improve performance. The standards focus on important patient, individual, or resident care and organization functions that are essential to providing safe, high quality care" (The Joint Commission 2016). This accomplishment provides the nursing staff with the confidence and assurance that our healthcare delivery is safe, effective and of the highest quality.

\section{References}

1. Knowles M. The Adult Learner: A Neglected Species. ERIC. 1973.

2. Magnussen L. Applying the Principles of Significant Learning in the e-Learning Environment. J Nurs Educ. 2008;47(2):82-86.

3. The Joint Commission. Comprehensive Accreditation Manual for Hospitals: Standards, Elements of Performance, Scoring and Accreditation Policies. Oak Brook, Illinois. Joint Commission Resources. 2016 